\title{
Alterstice
}

Revue internationale de la recherche interculturelle

International Journal of Intercultural Research

Revista International de la Investigacion Intercultural

\section{Métissage et hiérarchisation des cultures : les soubassements idéologiques de l'essentialisation des différences}

\section{Marika Moisseeff}

Volume 4, numéro 2, 2014

Santé mentale et sociétés plurielles

URI : https://id.erudit.org/iderudit/1077423ar

DOI : https://doi.org/10.7202/1077423ar

Aller au sommaire du numéro

Éditeur(s)

Alterstice

ISSN

1923-919X (numérique)

Découvrir la revue

Citer cet article

Moisseeff, M. (2014). Métissage et hiérarchisation des cultures : les

soubassements idéologiques de l'essentialisation des différences. Alterstice, 4(2), 25-37. https://doi.org/10.7202/1077423ar

\section{Résumé de l'article}

La discrimination des minorités visibles se fonde sur l'idéologie hiérarchisante qui conduit à appréhender leurs caractéristiques physiques en termes biologiques permettant de les situer à un palier inférieur sur l'échelle de l'évolution. Pour illustrer les répercussions tragiques de cette appréhension des différences, l'auteure prend pour exemple le sort qui a été réservé, en Australie, aux métis issus des relations entre les Aborigènes et leurs colonisateurs. 
ARTICLE THÉMATIQUE

\title{
Métissage et hiérarchisation des cultures : les soubassements idéologiques de l'essentialisation des différences
}

\author{
Marika Moisseeff ${ }^{1}$
}

\section{Résumé}

La discrimination des minorités visibles se fonde sur l'idéologie hiérarchisante qui conduit à appréhender leurs caractéristiques physiques en termes biologiques permettant de les situer à un palier inférieur sur l'échelle de l'évolution. Pour illustrer les répercussions tragiques de cette appréhension des différences, l'auteure prend pour exemple le sort qui a été réservé, en Australie, aux métis issus des relations entre les Aborigènes et leurs colonisateurs.

\section{Rattachement de l'auteure \\ ${ }^{1}$ CNRS Paris/Laboratoire d'anthropologie sociale, Paris, France}

\section{Correspondance}

marika.moisseeff@college-de-france.fr

\section{Mots clés}

Hiérarchisation des cultures; minorités visibles; Aborigènes australiens; métis; néoténie; évolutionnisme; théorie des climats; thérapie interculturelle

\section{Pour citer cet article}

Moisseeff, M. (2014). Métissage et hiérarchisation des cultures: les soubassements idéologiques de l'essentialisation des différences. Alterstice, 4(2), 25-38. 


\section{Introduction}

Dans leur présentation pour le colloque «Santé mentale et sociétés plurielles : de la différence culturelle à la diversité ", Betty Goguikian Ratcliff et llario Rossi ont suggéré que, par contraste avec les approches transculturelles, l'importance conférée aux différences culturelles risque de participer à leur essentialisation en générant " un sentiment de fossé infranchissable entre personnes de cultures différentes ». Certes, ce risque existe dès qu'il est question de décrire des faits ethnographiques mettant en évidence des distinctions fondamentales entre les représentations du monde et les modes de sociabilité occidentaux et ceux se rapportant à d'autres aires culturelles. Et cependant, ce sont bien ces distinctions, une fois documentées et explicitées dans toutes leurs complexités, qui permettront de rendre compte des mécompréhensions surgissant lors de certaines rencontres interculturelles. Or c'est précisément parce qu'il est très difficile de rendre compte des processus complexes de différenciation socioculturelle sans adopter une perspective comparative rigoureuse exigeant des descriptions ethnographiques minutieuses que la réduction des distinctions à des stéréotypes exotisants, tels que ceux véhiculés par les média, a le pouvoir de diffusion et la prégnance qu'on lui connaît. Mais si ces stéréotypes peuvent, de prime abord, sembler anodins, leurs conséquences ne le sont nullement, comme nous allons le voir. Dans cet article, j'irai même plus loin en suggérant que la prise en compte de leurs effets et de leurs fondements est parfois indispensable à la mise en place d'un cadre thérapeutique, dans la mesure où ils participent du ressenti relationnel lors d'une rencontre interculturelle (Moisseeff, 1999b).

Pour ce faire, plutôt que de m'attacher à décrire la spécificité d'une culture non occidentale ou d'une pratique thérapeutique particulière, je vais $m^{\prime}$ intéresser à l'idéologie occidentale qui sous-tend le processus de simplification de l'appréhension des différences et à quelques-unes de ses conséquences, actuelles et passées. Pour que l'on comprenne mieux ma démarche - c'est-à-dire la perspective décalée et volontairement généralisante pour laquelle on m'avait conviée à intervenir dans le colloque - il me paraît important de préciser quel en est le point de départ ${ }^{1}$. Je suis psychiatre et ethnologue. Mais la réflexion que je vais proposer ici est avant tout anthropologique. Elle se fonde sur la recherche que je poursuis depuis plus de trente ans sur les processus de constitution des identités, personnelles et collectives, notamment à partir d'un travail de terrain au long cours dans une communauté aborigène du sud de l'Australie (depuis 1992, voir Moisseeff 1997, 1999a, 2011b et 2014), mené parallèlement au développement d'une perspective culturelle comparative sur les représentations de la différence entre les sexes. Mon travail avec les Aborigènes australiens m'a conduit à être directement témoin d'actes racistes et à recueillir de nombreux récits s'y référant. Et ce, d'autant plus que les Aborigènes avec qui je travaille ont toujours eu à cœur de me les faire partager et qu'ils souhaitent que ces informations soient connues à l'extérieur de l'Australie. Par ailleurs, ma pratique clinique - distincte de celle de mes travaux universitaires en ethnologie - m'a amenée à exercer comme psychiatre et formateur en thérapie familiale systémique dans des départements et territoires français d'outre-mer (DOM et TOM) et des pays anciennement colonisés par la France. Les suggestions que je vais faire concernant la façon de poser un cadre thérapeutique lors d'une rencontre interculturelle, bien qu'elles ne constituent pas le cœur de mon propos, découlent de cette double pratique de chercheur en ethnologie et de clinicienne. Sur un plan plus théorique, elle me permet aussi de rapporter le processus d'essentialisation moins aux différences culturelles en tant que telles qu'à l'idéologie hiérarchisante occidentale ayant tendance à situer les populations à des niveaux distincts sur l'échelle de l'évolution. Mon analyse portera sur cet aspect précis de la culture occidentale. Elle est donc à la fois réflexive et, de ce point de vue, pleinement généralisante de façon assumée.

Une anecdote va me permettre d'introduire la problématique dont il va être question par la suite. II y a quelques années, alors que je mentionnais la visite, en France, d'amis aborigènes avec qui j'avais travaillé en Australie, une jeune femme me fit la réflexion cinglante suivante : "Quoi?! Vous les avez fait venir en France! Vous êtes des criminels! » Elle sous-entendait par là qu'en transplantant, ne serait-ce que pour quelques semaines, de supposés primitifs de l'âge de pierre dans " la civilisation ", mon mari et moi allions contribuer à les souiller, les transformer, leur ôter leur innocence originelle de bons sauvages. Dis-moi qui tu es, je te dirai où tu dois être et où tu dois demeurer pour que je puisse t'admirer comme je souhaite te rêver. Cette anecdote permet de repérer le racisme

\footnotetext{
${ }^{1}$ Je suis reconnaissante envers le lecteur anonyme qui m’a incitée à clarifier ma démarche.
} 
implicite qui sous-tend le mythe persistant du bon sauvage corrompu par la civilisation introduit par Rousseau au $18^{\mathrm{e}}$ siècle, c'est-à-dire à la suite de la découverte des Amériques et de l'Océanie et de la confrontation avec les populations qui y vivaient à l'écart de l'Europe jusqu'à leur colonisation. De fait, comme le rappelle Taylor, l'évolutionnisme anthropologique de la deuxième moitié $\mathrm{du} 19^{\mathrm{e}}$ siècle emprunte à la pensée des philosophes du $18^{\mathrm{e}}$ siècle " le postulat central d'une histoire universelle embrassant dans un même mouvement, orienté et non réversible, l'ensemble de l'humanité. Les sauvages sont alors convertis en primitifs, et leurs cultures deviennent des incarnations ou des figurations du passé des sociétés "civilisés" » (1991, p. 270-271). Cependant, il " se démarque des philosophies historicistes des Lumières par le poids qu'il assigne aux facteurs biologiques » et, par là à l'inégalité des "races", ce qui va ainsi permettre de "rendre compte de la stagnation culturelle des populations non occidentales » : " certaines sociétés ont progressé, tandis que d'autres paraissent figées dans une irrémédiable primitivité. " (p. 271). En effet, entre-temps l'évolutionnisme biologique a progressé, notamment en raison de la portée de la perspective darwinienne qui se diffuse dès la parution de L'Origine des espèces en 1859. II confère à la hiérarchisation historique des populations héritée des Lumières une dimension biologique qui a conduit, au cours du $19^{\mathrm{e}}$ siècle, à aborder les différences physiques à l'exemple des différences morphologiques permettant de classifier les espèces tout en leur assignant une place sur l'échelle de l'évolution (Moisseeff, 1999b). Les anthropologues ont depuis longtemps abandonné cette perspective, mais elle demeure encore très vivace dans les représentations populaires.

Je vais tenter de montrer que le racisme dont font l'objet les personnes ayant des traits physiques permettant de les repérer comme différentes de ceux des membres de la culture dominante occidentale dans laquelle elles vivent se fonde non sur leurs différences culturelles, mais sur le fait de les percevoir immédiatement comme inférieures parce qu'elles appartiendraient à un groupe humain censé être plus proche de l'animalité que ne le seraient les " vrais civilisés » qui, eux, se seraient éloignés de leur nature animale grâce à une intelligence supérieure leur ayant permis de contrôler la nature en développant leur technologie. Les Noirs, par exemple, tendent encore à être représentés comme le chaînon manquant entre le singe et l'homme, ce qu'illustrent les propos ignominieux dont sont la cible, aujourd'hui encore, les personnes de couleur ayant des postes éminents dans nos contrées occidentales.

\section{L'animalisation des minorités visibles}

En avril 2011, aux États-Unis, une responsable républicaine a fait circuler sur internet un montage photographique dans lequel le visage du président Obama avait été apposé sur la photo d'un petit chimpanzé (Clavel, 2013) de façon à le dépeindre comme l'enfant de deux chimpanzés adultes, ajoutant comme légende à ce photomontage "Maintenant vous savez pourquoi il n'a pas de certificat de naissance ". En dépit de la révocation de cette politicienne, ce genre de photomontages animalisant le président américain et sa femme n'a cessé depuis lors de se multiplier sur la Toile. En Italie, en juillet 2013, un sénateur de la Ligue du Nord a dit de la ministre de I'Intégration, originaire de la République démocratique du Congo, Cécile Kyenge : "Quand je vois les images de Kyenge, je ne peux m'empêcher de penser à des ressemblances avec un orang-outan ", tandis qu'un spectateur lui a lancé des bananes alors qu'elle prononçait un discours (cité par Clavel, 2013). En France, la ministre noire de la Justice, Christiane Taubira, a été à plusieurs reprises publiquement comparée à un singe tandis qu'un prêtre défilant contre le mariage homosexuel a crié dans un porte-voix " Y'a bon Banania, y'a pas bon Taubira " (cité par Huertas, 2013). Il en va de même sur Internet : "On dit que l'homme descend du singe, mais dans le cas de cette guenon ce doit être très récent ", a écrit, par exemple, une internaute (cité par Ndala, 2013). Dans un reportage diffusé le jeudi 17 octobre 2013 sur France 2, une candidate du Front national, invitée à commenter le photomontage comparant la ministre française à un singe qu'elle a diffusé sur son compte Facebook, a qualifié celle-ci de " sauvage ", rajoutant devant les caméras : " Je préfère la voir dans un arbre après les branches [sic] que de la voir au gouvernement " (Huffington Post, 2013). Des propos sur lesquels est revenu le présentateur noir français Harry Roselmack dans Le Monde du 4 novembre 2013 :

Ce qui me chagrine, c'est le fond de racisme qui résiste au temps et aux mots d'ordre, pas seulement au sein du FN, mais au plus profond de la société française. C'est un héritage des temps anciens, une justification pour une domination suprême et criminelle : l'esclavage et la colonisation. [...] Combien de fois ai-je dû expliquer à un restaurateur ou même à un camarade que les vieilles affiches "Y'a bon Banania" qu'ils accrochent à leurs murs ne peuvent pas être regardées qu'avec amusement 
ou nostalgie. Comme certains albums de bande dessinée qui ont égayé notre enfance, elles laissent des empreintes d'un autre temps dans nos imaginaires.

Tout le monde " sait » que le racisme existe, et les professionnels de la psychothérapie interculturelle sont, a priori, particulièrement bien placés pour le savoir. Si j'ai choisi de citer cet ensemble de données, au risque de lasser et de générer du malaise, c'est pour insister sur le fait que l'essentialisation renvoie non seulement à des représentations qui s'attachent à l'apparence physique en l'attaquant tout en lui donnant corps, mais plus profondément encore qu'elle engendre un vécu émotionnel lui aussi très incarné, celui précisément évoqué par Harry Roselmack. Pour être des thérapeutes compétents, c'est-à-dire pour paraphraser Winnicott, suffisamment bons, il nous faut, j'en suis convaincue, être à même en certaines circonstances de faire l'expérience de ce malaise (et non simplement d'en être conscients) et en prendre acte. Cela n'est certes pas suffisant pour être efficace mais peut, de mon point de vue, en être la condition indispensable, en permettant l'établissement d'une relation de confiance et d'intercompréhension lorsque le contexte interculturel en jeu est pipé par des relations éminemment asymétriques. L'attrait pour l'exotisme et le désir de connaissance qu'il est susceptible d'inspirer est souvent à l'origine légitime des vocations des ethnologues et de certains ethnopsychiatres. II m'est cependant arrivé de rencontrer des cliniciens chez qui la fascination pour l'anthropologie tendait malheureusement à prendre le pas sur leur capacité à reconnaître l'importance de la discrimination dont leurs patients étaient l'objet et qui participait pourtant de la souffrance de ces derniers à voir leur culture ou celle de leurs parents dévalorisée. II ne s'agit pas, bien entendu, de prétendre occuper la place de nos patients mais de prendre celle de témoin, car au-delà du savoir distancié sur la culture de l'autre, ce qui importe véritablement dans certaines thérapies interculturelles, c'est d'accepter d'être affecté. C'est peut-être le tout petit prix à payer pour faire un pas vers le ressenti intérieur des victimes du racisme ${ }^{2}$. Eux, n'ont, en effet, pas le luxe de mettre à distance le miroir déformant qu'on leur tend quotidiennement.

Je reprendrai, pour illustrer cette proposition, l'exemple de la visite, en France, de mes amis aborigènes australiens. Alors qu'ils étaient installés à la terrasse d'un café parisien, ils nous firent part avec un grand émoi du confort qu'ils ressentaient à faire enfin l'expérience de l'invisibilité, invisibilité qui ne leur est jamais accordée en Australie. Làbas, leur apparence physique les désigne d'emblée comme Aborigènes pour les Blancs, et métis pour les Aborigènes, ce qui surdétermine leurs relations aux autres en leur imposant une vigilance, une conscience de soi particulièrement contraignante. En revanche, dans une grande capitale européenne cosmopolite comme Paris, nul ne pouvait préjuger avec certitude de leur origine et personne d'ailleurs n'y prêtait attention, ce qui leur conférait une immunité ethnique temporaire qu'ils appréciaient énormément et qu'ils ressentaient comme une véritable libération.

De fait, dans la plupart des pays européens, être blanc confère une invisibilité confortable dont les Blancs sont en général peu conscients. En dehors des circonstances où ils choisissent de se plonger volontairement dans un bain exotique coloré, par exemple lors de voyages touristiques outremer, l'anonymat est un luxe qui leur est réservé. Ce privilège est, au contraire, communément refusé aux membres des minorités ethniques lorsqu'ils vivent au sein d'une société où ils sont repérés comme migrants, descendants d'immigrés ou d'esclaves, indigènes ou métis.

Des divers exemples que je viens d'évoquer, il ressort que le racisme dont sont la cible les minorités visibles se fonde moins sur la réalité de la particularité de leur culture que sur l'association de leur apparence à une infériorité fondamentale renvoyant à une asymétrie des relations entre ex-colonisateurs et ex-colonisés. La dépréciation de leur culture est aisément mise au compte de leur phénotype, c'est-à-dire d'un type racial distinct, ostentatoire et immédiatement rapporté à une infériorité biologique : ils sont censés renvoyer au passé des peuples civilisés dont le paradigme est la société occidentale et, plus spécifiquement aujourd'hui, la société nord-américaine. Soyons clairs : si les traits physiques interviennent comme facteurs de hiérarchisation entre cultures, c'est qu'ils ont été investis par la pensée évolutionniste occidentale du $19^{\mathrm{e}}$ siècle qui les a rattachés à des différences biologiques et, par là, à une nature immuable. De fait, une géographie imaginaire particulièrement prégnante situe l'avant-garde de l'évolution humaine et socioculturelle au Nord et à l'Ouest.

\footnotetext{
${ }^{2}$ Les Aborigènes expriment volontiers ce souhait que les Blancs - les Aborigènes sont noirs de peau et désignés comme tels, "black fellows » - prennent conscience de l'expérience du racisme qu'ils leur font subir.
} 


\section{Les points cardinaux de l'évolutionnisme}

L'Est est le lieu imaginaire d'où sont censées être venues les hordes de Barbares dont les Occidentaux seraient les descendants lointains. L'axe Est-Ouest tend donc à renvoyer au passage de la barbarie à la civilisation. L'Ouest, c'est le point de l’horizon qui désigne une société à la fois démocratique et technologiquement prométhéenne. L'axe Nord-Sud, quant à lui, renvoie au passage progressif de l'animal à l'humain, c'est l'axe de l'humanisation rattachée au blanchiment progressif de l'humanité (Moisseeff, 2010). La noirceur de la peau est, comme nous venons de le voir, aisément assimilée à la substitution du pelage recouvrant les primates qui nous ont précédés, et à la " sauvagerie » des cultures du Sud - africaines et latines. On a donc affaire à une géographie symbolique dessinant les lignes "évolutives» de populations physiquement et, de manière corrélative, "mentalement » distinguées, ce qui permet de hiérarchiser automatiquement leurs cultures respectives.

Ainsi le généticien américain James Watson, prix Nobel de médecine en 1962 en tant que codécouvreur de la structure de I'ADN, a déclaré dans une entrevue publiée le 14 octobre 2007 dans le Sunday Times qu'il était " profondément pessimiste quant à l'avenir de l'Afrique » car « toutes nos politiques sociales se fondent sur le fait que leur intelligence est la même que la nôtre [Occidentaux blancs], alors [...] que toutes les recherches concluent que ce n'est pas vraiment le cas ". Il a ajouté que, bien qu'il soit naturel de désirer que tous les êtres humains soient égaux, « ceux qui ont affaire à des employés noirs savent que ce n'est pas vrai » (cité par Milmo, 2007).

Dans les médias, l'opposition entre le Nord et le Sud, l'Est et l'Ouest expriment les distinctions que nous établissons quasi naturellement entre sociétés "développées ", " en voie de développement " ou " sous-développées ", ou encore «du tiers-monde". Convenons que ces appellations masquent avec plus ou moins d'efficacité la perpétuation d'une hiérarchisation des cultures. De fait, cette géographie symbolique poursuit le processus idéologique d'essentialisation des groupes humains qui, de l'Antiquité à nos jours, en passant par des penseurs aussi éminents qu'Aristote ou Montesquieu, repose sur la théorie des climats qui, comme l'ont montré Gourou (1963) et Bourdieu (1980), continue, à notre insu le plus souvent, à œuvrer de nos jours. Selon cette théorie, le climat froid et rude des régions septentrionales opposé à celui chaud et humide des régions méridionales permet de rendre compte de la supériorité des " races " nordiques, rationnelles, dures et " naturellement " dominatrices et, à l'inverse, de l'infériorité des « races » méridionales, émotionnellement chaudes et molles et, par là, vouées à l'asservissement.

Cette vieille théorie des climats a été par la suite subrepticement intégrée à une perspective évolutionniste de I'histoire humaine, elle-même calquée sur l'évolution des espèces proposée par Darwin, et selon laquelle toutes les sociétés humaines passeraient par les mêmes stades - sauvagerie, barbarie et civilisation -, certaines étant perçues comme bloquées à un stade antérieur (Moisseeff, 1999a). L'idéologie racialiste, en intégrant la perspective évolutionniste à la théorie des climats, va permettre de légitimer l'articulation entre l'apparence physique des membres de certains groupes humains et leur degré d'évolution tout en la faisant correspondre à la géographie symbolique extrêmement prégnante que je viens d'évoquer : les " races " supérieures, situées au Nord, sont blanches, les " races " inférieures du Sud sont brunes, voire noires, une pigmentation foncée associée, comme nous l'avons vu, au pelage de l'espèce animale, simiesque, qui les précéderait immédiatement sur l'échelle de l'évolution et dont les races nordiques se seraient, elles, morphologiquement et sociologiquement éloignées depuis longtemps.

Cette association, sur le plan des représentations socioculturelles occidentales, entre la noirceur de la peau et le pelage animal doit être, selon moi, mise sur le compte d'une autre théorie qui infiltre l'idéologie raciale contemporaine. Il s'agit de la théorie de la fœtalisation de l'espèce humaine proposée, dans les années 1920, par Bolk (1926/1961), plus connue sous l’appellation de théorie de la néoténie. Pour Bolk, la particularité de la morphologie du corps humain - dépigmentation, absence de pelage, boîte crânienne ovoïde contenant un gros cerveau, visage d'aspect juvénile par réduction de la taille des mâchoires, des dents, des arcades sourcilières et de la projection nasale - serait la résultante de l'apparition, chez l'homme, d'un facteur inhibant la différenciation des structures morphologiques en sorte qu'elles n'évoluent pas vers un stade adulte achevé comme chez les autres mammifères. D'une part, l'homme naîtrait néotène, c'est-à-dire avant terme, et la fermeture tardive consécutive des jointures des os de son crâne, des fontanelles, aurait permis la poursuite de son développement cérébral et de 
ses capacités intellectuelles afférentes bien au-delà de celui des autres primates. D'autre part, il conserverait, une fois devenu adulte, les caractéristiques physiques propres aux fœtus des primates.

La spécificité de l'aspect physique de l'être humain va donc pouvoir être rattachée à sa supériorité vis-à-vis des autres espèces, ce qui l'aurait conduit à dominer la nature et à se hisser à la cime de l'échelle de l'évolution grâce à l'essor constant de sa technologie. Dans cette perspective, " les caractéristiques juvéniles [humaines] sont un réservoir d'adaptations potentielles » (Gould 1977/1997, p. 70; voir aussi Moisseeff, 2008b). Comme nous allons le voir, la cohérence de ce schème explicatif associant supériorité ou infériorité de certaines espèces, et corrélativement leur plus ou moins grande "ancienneté ", au degré de fœtalisation de leur morphologie va permettre de l'appliquer à la classification des « races».

Remarquons tout d'abord combien la proposition selon laquelle le maintien d'une virtualité adaptative extrême, grâce à l'absence de différenciation et à la conservation de caractéristiques quasi " embryologiques ", rejoint le discours actuel sur les cellules souches : moins un organisme ou un de ses éléments est différencié, plus il est perçu comme porteur d'un devenir infiniment démultiplié. Cette idée a sans doute conduit à dépeindre les espèces ou " races » censées dépasser l'homme sur le plan de l'intelligence comme des fœtus : les extra-terrestres voyageant d'une galaxie à l'autre ont fréquemment une grosse tête ovoïde, de grands yeux et un corps petit ou longiligne, à l'instar de E.T., l'extraterrestre de Spielberg, ou des Asgards de la série américaine Stargate.

Et effectivement, le fait que nous puissions nous représenter " les formes infantiles de nos ancêtres » devait, selon Bolk, nous aider à concevoir la structure formelle de l'espèce qui nous succéderait. Mais, demande-t-il alors, "Qui s'aventurera à esquisser, partant des traits d'un nourrisson, le portrait futur de l'adulte ? » (1926/1961, p. 278-279). J'ai tenté de montrer ailleurs (Moisseeff, 2010) que les maîtres d'œuvres de la science-fiction contemporaine s'y étaient justement appliqués. Ils nous conduisent à imaginer les enfants de la nouvelle espèce comme des enfants blancs, blonds aux yeux bleus, dotés d'une intelligence extraordinaire bien supérieure à celle des humains actuels ${ }^{3}$. II est clair que ce qui sous-tend l'apparence donnée à ces enfants procède de l'intégration de la théorie de la néoténie à la mythologie occidentale contemporaine (Moisseeff, 2005b). En effet, selon Bolk, la forme des espèces qui se succèdent au cours de l'évolution acquiert un aspect fœtal toujours plus affirmé (1926/1961, p. 249). Nous pouvons maintenant comprendre l'apparence donnée aux enfants de la nouvelle espèce humaine par les auteurs et réalisateurs de science-fiction : elle renvoie au renforcement de leur fœtalisation qui s'exprime par la dépigmentation marquée de leur peau, de leurs cheveux et de leurs yeux que les races nordiques auraient été, selon le père de la néoténie, les premières à revêtir.

Par ailleurs, la fœtalisation de l'homme aurait aussi, selon Bolk, fortement retardé l'âge auquel il accède à sa fonction reproductrice : l'accession à la reproduction ne constitue plus la raison d'être première de la maturation des individus, comme c'est habituellement le cas dans les autres espèces inférieures, qui sont beaucoup plus prolifiques que lui. Et la science-fiction décrit effectivement, avec une certaine constance, la nouvelle espèce humaine censée succéder à Homo sapiens sapiens, comme hypofertile ou stérile (Moisseeff, 2008b, 2010 et 2011a). Darwin, dans son autobiographie (Darwin et Barlow, 1958), reconnaît sa dette envers Malthus. Or, selon un point de vue malthusien simplifié, les populations jugées inférieures, qu'il s'agisse de classes sociales ou de races, sont censées être plus prolifiques que les catégories supérieures. Bolk, pour sa part, comme je l'ai dit, rend compte de la diminution de la fertilité de l'espèce humaine par le processus de sa fœtalisation qui freinerait son accession à la fonction reproductrice tout en lui permettant de la réprimer fortement au profit d'autres activités sociales. Mais, comme nous allons le voir, il ne pense pas que toutes les populations humaines aient atteint un égal degré d'évolution. Sa perspective va ainsi pouvoir venir conforter l'idée néo-malthusienne selon laquelle les populations les plus prolifiques seraient également les plus ancrées dans l'animalité (Moisseeff, 2000, 2004 et 2008a).

Dans l'Occident contemporain, l'appréhension des différences culturelles est sous-tendue par l'ensemble des théories que je viens d'évoquer et qui, toutes, convergent dans le même sens : les populations du Sud et de l'Est

\footnotetext{
${ }^{3}$ Sur le thème de l'apparition d'enfants appartenant à une espèce humaine supérieure à l'espèce homo sapiens sapiens actuelle, on se reportera, par exemple, pour les livres, à Wyndham (1957), Cook (1989), Rice (1993/1995, 1994/1996), Bear (1999/2001, 2003), pour les films, Le village des damnés (Rilla, 1960; Carpenter, 1995), Godsend (Beaumont, 1980).
} 
dont sont issus les migrants les plus pauvres sont censées être restées, à l'instar des animaux, inféodées aux nécessités de la reproduction, ce qui rendrait compte de leur fort taux de fécondité, aux dépens de la valeur accordée à l'érotisme et à la libération des femmes, toutes choses qu'ont pu promouvoir les sociétés plus évoluées du Nord et de l'Ouest grâce à l'essor de la technologie qu'elles ont su impulser et qui leur a permis de maîtriser leur reproduction.

Mais c'est bien la théorie de la néoténie qui va permettre d'articuler cette appréhension des différences culturelles à la discrimination raciale fondée sur le phénotype. Selon Bolk, en effet, la " race noire " ne se serait pas encore pleinement dégagée de la morphologie animale de l'espèce qui l'a précédée, ce qui rendrait compte de son infériorité exprimée dans son apparence :

Que je sois, sur la base de ma théorie, un partisan convaincu de l'inégalité des races, cela est facile à comprendre. Toutes les races n'ont pas avec le même degré progressé sur le chemin de l'évolution humaine. [...] Si maintenant dans l'avenir, chez la race nègre la fœtalisation et le retardement [de la maturité] s'affirment de façon progressive, alors la voie lui est ouverte pour atteindre le degré d'évolution et de structure des plus hautes races. (Bolk, 1926-1961, p. 273-274)

Selon Bolk, donc, les races blanches, blondes et aux yeux bleus ont atteint le stade le plus achevé de l'évolution biologique actuelle de l'espèce humaine. Et c'est sans doute une perspective analogue qui a incité le généticien James Watson à proférer les propos scandaleux cités plus haut.

L'affirmation politiquement correcte actuelle selon laquelle les races n'existent pas, aussi humaniste et scientifiquement fondée qu'elle soit, tend à nier les répercussions de la stigmatisation des individus fondée sur l'appréhension de leur particularité morphologique ${ }^{4}$. Le nier participe, bien qu'involontairement le plus souvent, à la dénégation des traumatismes qu'ils vivent de façon répétée au quotidien. La hiérarchisation des cultures reste sous-tendue par une hiérarchisation racialisante sociocuturellement construite des différences d'apparence physique d'autant plus insidieuse qu'elle est réfutée par ceux qui ne sont pas susceptibles d'en souffrir et qui pourtant en sont les instruments, comme nous le rappelle Roselmack. Cette réfutation systématique conduit à la méconnaissance des drames que génère la hiérarchisation, ce dont témoigne particulièrement bien la problématique de ceux qui sont qualifiés de métis. Ce terme est, en effet, appliqué, en général, non pas à l'ensemble des individus ayant des ascendants affiliés à des cultures distinctes, mais uniquement à ceux dont les "cultures » renvoient à des populations aux phénotypes caractéristiques. Le corps apparaît alors comme un indicateur ineffaçable de discrimination que l'on rapportera immédiatement à l'essence intrinsèque des individus concernés. Et quand il existe une relation hiérarchique entre cultures, tout se passe comme s'il était impossible de vivre paisiblement son métissage. On est sommé de choisir entre ses deux communautés d'appartenance, et ce choix nécessaire confine au sentiment de perte (Moisseeff, 2005a et 2013). Une amie australienne non aborigène qui avait eu des enfants avec un Aborigène me dit un jour : je les laisserai choisir. Comme s'il fallait effectivement choisir l'une ou l'autre plutôt que l'une et l'autre de ses affiliations.

L'idéologie racialiste dont je viens de brosser à gros traits l'histoire des soubassements a, en effet, une incidence tout à fait patente sur ce qu'ont à vivre aujourd'hui encore les ressortissants de pays ayant été colonisés par ceux qui se voyaient comme appartenant à des races supérieures, justifiant, par là, leur mission civilisatrice.

\section{Le métis comme révélateur}

À la fin du $18^{\mathrm{e}}$ siècle, lorsque les Britanniques abordent les rivages du continent australien pour en prendre possession, la population face à laquelle ils se trouvent confrontés leur paraît renvoyer au degré zéro de I'humanité. De fait, tous les critères scientifiques allaient bientôt converger pour les reléguer au statut de reliquat d'une population préhistorique, plus proche de l'homme de Néandertal que de Cro-Magnon. Comme l'écrit un anthropologue australien : " Les éléments très archaïques de leur type racial ont pu faire supposer une relation avec l'homme du paléolithique supérieur (et même, selon certains, du paléolithique moyen) » (Elkin, 1978, p. 831).

\footnotetext{
${ }^{4}$ II y a une vingtaine d'années, un slogan antiraciste australien proclamant " Different colors, one people " avait entraîné la réprobation des Aborigènes qui tenaient, en raison du sort qui leur avait été réservé au cours de la colonisation, à maintenir « Different colors, different peoples».
} 
Outre le teint foncé et l'abondance du système pileux, d'autres traits viennent conforter l'idée d'un type racial peu évolué : «Presque dolichocéphale [c'est-à-dire ayant le crâne allongé], l'Aborigène australien a le front fuyant, de profondes cavités temporales, les yeux très enfoncés, la racine du nez fortement en retrait sur des arcades sourcilières proéminentes. La base du nez est aplatie et évasée, la bouche large, les lèvres habituellement épaisses mais non éversées, le prognathisme [c'est-à-dire la projection en avant des mâchoires] est commun. » (p. 831) On peut, dès lors, mettre sur le compte de l'archaïsme de la " race » aborigène les caractéristiques de leur culture : économie de chasse et de cueillette, outils très peu nombreux, absence de vêtements et d'habitats permanents. Or le critère de développement technologique est, du point de vue occidental, le critère fondamental utilisé pour jauger du degré d'évolution des cultures. Il est, en effet, beaucoup plus facile à évaluer que ne le serait celui du degré de sophistication des systèmes de parenté ou des métaphysiques locales. La population autochtone australienne fut donc considérée inapte à concevoir une quelconque notion de propriété foncière. En conséquence, aucun traité ne fut établi comme il était de règle ailleurs en des circonstances analogues. Le continent australien fut déclaré terra nullius, c'est-à-dire " terre n'appartenant à personne ", et les Aborigènes furent l'un des seuls peuples à n'avoir obtenu aucune compensation en échange de la prise de possession de leurs territoires par la Couronne britannique.

Cette appréhension d'une distinction raciale foncière entre les Aborigènes et leurs colonisateurs britanniques a eu d'autres conséquences tragiques. Je n'aborderai ici que l'une d'entre elles : le sort réservé aux enfants métis. En effet, à la fin du $19^{\mathrm{e}}$ siècle, après un siècle de colonisation du continent australien par les Britanniques, la population des Aborigènes dits de sang pur a diminué à un point tel que cela incite à penser qu'ils vont disparaître. Par contre, l'augmentation constante, en dépit de l'interdiction des relations sexuelles entre Blancs et Noirs, de la population métisse devient un sujet de préoccupation majeur pour les instances gouvernementales qui cherchent à promouvoir l'établissement d'une nation australienne blanche. C'est dans ce but que des régulations très restrictives concernant l'entrée des immigrants vont être mises en place (Bartrop, 2001). Mais les indigènes d'Australie ne sont pas des migrants et il est impossible de les expulser. On va alors promulguer des lois visant à faire disparaitre les caractéristiques physiques et culturelles de la population native australienne (Bartrop, 2001; Neville, 1947; Tatz, 1999). La finalité est, comme le dit un Protecteur en chef des Aborigènes, de pouvoir oublier qu'a existé un jour une population aborigène en terre australienne. On confère donc aux bien mal nommés protecteurs des Aborigènes le pouvoir d'utiliser la force publique pour séparer les enfants métis de leurs familles. Voici, en guise d'exemple, ce que déclara en 1909 le protecteur en chef des Aborigènes de l'État de l'Ouest de l'Australie :

Le métis est intellectuellement supérieur à l'Aborigène et il en va du devoir de l'État qu'il leur soit donné une chance de vivre une vie meilleure et plus pure que celle de leurs frères. Je n'hésiterais pas un instant à séparer tout métis de sa mère aborigène, quelle que soit l'ampleur de la démonstration de sa détresse au moment de la séparation. Elles oublient vite leurs enfants. (cité dans Commonwealth of Australia, 1997, p. 276).

Les métis sont supérieurs à leurs ascendants aborigènes car le sang blanc qui coule en eux les élève à une condition un peu supérieure à celle des Noirs de " race pure ». On estime, en conséquence, qu'il est absolument nécessaire de les soustraire à l'influence néfaste de leurs familles aborigènes pour que s'exprime cette parcelle d'intelligence qu'ils recèlent en eux. Toutefois, les métis demeureront inférieurs aux Européens tant que leurs caractéristiques proprement aborigènes n'auront pas disparu. II leur est donc interdit de se marier avec plus noirs qu'eux. La police patrouille tout le pays pour arracher les enfants métis à leurs parents afin de les donner en adoption à des familles blanches ou de les placer dans des institutions situées à des milliers de kilomètres de leur communauté d'origine. II est interdit à ces enfants d'avoir le moindre contact avec leurs parents aborigènes ou de parler dans leur langue maternelle. Tout est fait pour qu'ils oublient ce qui leur a été légué par leur famille d'origine. Ces mesures, appliquées jusqu'au début des années 1970, ont eu des conséquences absolument dramatiques, non seulement pour les dizaines de milliers d'individus enlevés - ceux que l'on désigne aujourd'hui en Australie comme les générations volées -, mais également pour leurs familles et leurs communautés.

Les Aborigènes se sont battus pendant des décennies pour que le gouvernement reconnaisse les préjudices irréparables qu'ils ont subis. Le 13 février 2008, ce jour est enfin venu et il restera à jamais gravé dans la mémoire des Australiens. Ce jour-là, tous, y compris les enfants qui étaient à l'école, ont arrêté leurs activités habituelles 
pour regarder sur les écrans de télévision les excuses que Kevin Rudd, le premier ministre, a adressées aux peuples indigènes de l'Australie.

\section{Excuses officielles et officieuses}

Voici une partie de ma traduction du texte qu'il a prononcé :

Nous sommes là aujourd'hui pour rendre hommage aux peuples indigènes de cette terre.

Nous nous remémorons les mauvais traitements qui leur ont été infligés dans le passé.

Nous nous remémorons en particulier les mauvais traitements infligés aux générations volées, ce chapitre entaché de notre histoire nationale.

Nous nous excusons pour les lois et les mesures politiques mises en place qui ont infligé tant de douleur, de souffrances et de préjudices à nos compatriotes.

Nous nous excusons surtout pour avoir retiré à leur famille, à leur communauté et de leur terre natale les enfants aborigènes.

Nous demandons pardon pour la douleur, la souffrance et les blessures infligées à ces générations volées et à leurs descendants, et pour la désolation infligée à leurs familles.

Nous demandons pardon aux mères et aux pères, aux frères et aux soeurs, pour avoir causé le démantèlement de leurs familles et de leurs communautés.

Nous, Parlement de l'Australie, demandons avec tout le respect qui s'impose que ces excuses soient reçues de telle façon qu'elles participent au processus de guérison de la Nation.

II vient un moment dans l'histoire des Nations où leurs peuples doivent pouvoir se réconcilier avec leur passé.

Ce temps est venu pour l'Australie. Et c'est à cette fin que le parlement est aujourd'hui réuni : pour régler ce conflit irrésolu entre les peuples composant la nation et ainsi éliminer cette tache qui souille l'âme nationale.

Aux générations volées, je dis la chose suivante:

En tant que premier ministre de l'Australie, je vous demande pardon.

Au nom du gouvernement de l'Australie, je vous demande pardon.

Au nom du Parlement de l'Australie, je vous demande pardon.

Et je vous offre ces excuses sans aucune réserve.

Nous nous excusons pour les actes infamants, les dommages et les humiliations que ces lois ont entraînés.

Lors du discours du premier ministre, un grand nombre d'Aborigènes se trouvaient dans la salle du parlement où ils avaient été, pour la première fois, conviés, d'autres venus de tout le pays étaient installés sur les pelouses faisant face au parlement. Je vous invite à regarder leurs réactions telles qu'elles ont été filmées ${ }^{5}$. Elles nous permettent, en effet, de ressentir, à l'unisson de la leur, leur poignante émotion. Faire cette expérience est, de mon point de vue, un privilège et non du voyeurisme. C'est un petit pas pour éprouver de l'intérieur l'expérience des sujets qui sont discriminés en raison de leurs différences.

\footnotetext{
${ }^{5}$ http://www.youtube.com/watch?v=DUvPxmiudSI\&feature=player_embedded
} 
Et, pourtant, on s'en doute, un tel événement a attisé, en proportion de son exceptionnalité, la haine d'un certain nombre de racistes australiens. Pour illustrer la persistance du regard évolutionniste qu'ils portent sur la communauté aborigène, voici donc maintenant une partie des pseudo-excuses qui ont circulé sur internet juste avant que le premier ministre n'adresse ses excuses officielles (Australian News Commentary, 2008):

Nous nous excusons pour vous avoir donné des docteurs et des soins médicaux gratuits, ce qui vous a permis de survivre et de vous multiplier en sorte que vous puissiez exiger de nous des excuses.

Nous nous excusons pour vous avoir aidé à lire et vous avoir appris l'anglais, vous ouvrant ainsi les portes de l'ensemble de la civilisation, de la pensée et de l'esprit d'entreprise.

Nous nous excusons pour avoir construit de grandes fermes et de grandes propriétés foncières, qui permettent aujourd'hui de vous nourrir alors qu'auparavant vous viviez de ce que la terre vous fournissait et que vous mourriez de faim en cas de sécheresse.

Nous nous excusons pour vous avoir fourni des vêtements chauds faits de matériaux tissés qui ont remplacé les peaux d'animaux que vous utilisiez auparavant.

Nous nous excusons pour avoir travaillé dur afin de payer des impôts qui financeront vos aides sociales, votre couverture médicale, votre éducation, etc.

Nous sommes seulement trop heureux de reprendre tout ce qui est cité ci-dessus et de vous renvoyer dans votre brousse quand vous vous en sentirez prêts.

Ce texte est un exemple de la manière dont on rappelle sans cesse aux Aborigènes qu'ils ne sont que des primitifs dépendant à tout jamais de la culture blanche pour leur survie. Pas moyen de ce point de vue de les considérer comme des égaux. Nous voyons donc que les différences culturelles entre Aborigènes et non Aborigènes continuent à être mises au compte de natures sous-jacentes foncièrement et irrémédiablement distinctives. Dans cette perspective, "s'ils veulent vivre avec nous ", ils doivent s'assimiler, c'est-à-dire disparaître en tant que groupe culturel distinctif. En 1983, un magnat de l'industrie minière australienne déclara que les métis qui n'arrivaient pas à s'intégrer à la civilisation posaient un sérieux problème, et que s'il ne tenait qu'à lui de le résoudre, il ferait en sorte qu'ils ne puissent plus se reproduire en introduisant des substances stérilisantes dans leur eau (O'Rourke, 1984).

\section{De la nécessité d'être affecté}

Je voudrais cependant suggérer ici que ces " antimigrants " que sont les membres des populations autochtones partagent des aléas existentiels similaires à ceux des migrants, qui subissent eux aussi les conséquences d'une colonisation antérieure, ceux-là mêmes qui sont reçus en consultation ethnopsychiatrique. Le problème fondamental auquel ces minorités sont confrontées est moins le déplacement ou les différences culturelles que la relation hiérarchique existant entre leur culture d'origine et la culture dominante de leurs ex-colonisateurs. Car, soyons clairs, ces consultations ne s'adressent généralement pas à tous les migrants. II ne viendrait pas à l'idée de mon mari américain vivant en France ou d'un chef d'entreprise japonais d'une filiale internationale de s'adresser à un ethnopsychiatre. Tout se passe, en effet, souvent - et on peut certes le regretter - comme si ces consultations concernaient avant tout les membres des communautés précarisées en raison de leur origine socioculturelle.

Qu'en conclure pour la clinique? Je voudrais suggérer ici que pour mettre en place une relation de confiance avec les patients qui consultent dans les services de psychiatrie interculturelle, il est nécessaire d'être conscient de ce dont ils sont eux-mêmes conscients, à savoir que leurs cultures, pour exotiques qu'elles puissent être considérées, tendent à être jugées comme archaïques. On admet ainsi, en général, que les sociétés occidentales

${ }^{6}$ Du point de vue des populations autochtones, par exemple amérindiennes ou australiennes, les personnes issues de l'immigration sont celles venues des autres continents et renvoient d'abord à leurs colonisateurs européens qui les ont, selon leurs termes, " envahies » et colonisées. Et, de fait, l'émigration des populations autochtones actuelles vers d'autres pays est numériquement négligeable, notamment en regard de l'émigration massive des Européens au cours du $19^{\mathrm{e}}$ siècle. 
contemporaines renvoient à la culture la plus civilisée. On en prend pour preuve - et les médias ne s'en privent pas - la déclaration selon laquelle c'est en Occident que la démocratie serait la plus aboutie : tous les citoyens y sont considérés comme égaux en droit et prétendument traités en conséquence. La preuve ultime en est rapportée au fait que " la femme " y serait pleinement traitée à l'égale de l'homme, et qu'elle y a accès à la liberté sexuelle, à la contraception et à l'avortement. Inversement, les hommes des sociétés jugées plus " arriérées " sont de façon commune perçus comme des tyrans domestiques s'acharnant à limiter la liberté de leurs épouses et de leurs filles.

Les ressortissants des pays dits en voie de développement ou d'ex-colonies ont internalisé l'appréhension dévalorisante et dégradante de leur culture au travers de la perception d'eux-mêmes qui leur est régulièrement renvoyée ${ }^{7}$. Le premier ministre australien nous a, à juste titre, rappelé que la reconnaissance authentique, $c^{\prime}$ est-àdire manifestement ressentie au plan national, des préjudices dévastateurs du racisme était un préalable indispensable à la réconciliation entre des communautés ayant entretenu des rapports foncièrement inégalitaires et, de ce fait, catastrophiques dans leurs effets à long terme. Et il est un fait que des excuses officielles étaient réclamées depuis des décennies par les Aborigènes. Que les instances gouvernementales aient enfin accepté de les présenter situe dorénavant les Aborigènes comme des acteurs politiques à part entière et en tant que tels reconnus aptes à exercer les plus hautes fonctions dans leur propre pays. II est néanmoins évident que le passé et ses conséquences ne sont pas pour autant effacés. Cependant, on peut faire l'hypothèse que cette étape constitue la condition sine qua non du traitement des retentissements individuels et familiaux de cette histoire tragique.

Au terme de cette analyse des soubassements idéologiques occidentaux de l'essentialisation des différences culturelles et de quelques-unes de leurs conséquences, je proposerai, en guise de conclusion, de considérer que l'un des enjeux majeurs de la thérapie avec les représentants des minorités que j'ai évoquées doit consister à accepter d'être affecté par leur expérience continue de sujets dépréciés à qui est refusée l'aptitude à se fondre dans l'anonymat et l'indifférence. Être affecté, comme on doit l'être lorsqu'on accueille des victimes de violences extrêmes (Sironi, 2007; Waintrater 2003/2011). Les traumatismes itératifs consécutifs aux expériences quotidiennes de dépréciation et à leur internalisation doivent être accueillis avec le même respect et la même attention. Car, selon moi, maintenir sa capacité à être affecté et à transformer l'affect partagé en ressources thérapeutiques est à la base de tout acte thérapeutique digne de ce nom.

\section{Bibliographie}

Australian News Commentary. (2008, février). Apology to the Aboriginal population from mainstream Australia [en ligne]. http://www.australian-news.net/Aboriginal_apology.htm (consulté le 6 mai 2014)

Bartrop, P. (2001). The Holocaust, the Aborigines, and the bureaucracy of destruction : an Australian dimension of genocide. Journal of Genocide Research, 3(1), 75-87.

Bear, G. (1999/2001). L'Echelle de Darwin. Paris : Laffont.

Bear, G. (2003). Les Enfants de Darwin. Paris : Laffont.

Bolk, L. (1926/1961). Le problème de la genèse humaine. Revue française de psychanalyse, 25(2), 243-279.

Bourdieu, P. (1980). Le nord et le midi : contribution à une analyse de l'effet Montesquieu. Actes de la recherche en sciences sociales, 35, 21-25.

Clavel, G. (2013, 18 octobre). Singe, esclave, indépendantiste... Christiane Taubira cible de toutes les droites (en ligne). Huffington Post. http://www.huffingtonpost.fr/2013/10/18/singe-taubira-king-kong-cibledroites_n_4120910.html?utm_hp_ref=christiane-taubira

Commonwealth of Australia. (1997). Bringing Them Home: Report of the National Inquiry into the Separation of Aboriginal and Torres Strait Islander Children from Their Families. Sydney, Australie : Human Rights and Equal Opportunity Commission.

\footnotetext{
${ }^{7}$ Et, alors que je relis ces lignes en ce début janvier 2015 marqué, en France, par des actes terroristes perpétrés par de jeunes hommes " issus de l'immigration », je ne peux m'empêcher de penser que ce constat, s'il est insuffisant à tout expliquer de leurs gestes, y contribue au moins en partie.
} 
Cook, R. (1989). Mutation. Paris : Sylvie Messinger.

Darwin, C. et Barlow, N. (1958). The Autobiography of Charles Darwin 1809-1882. With the original omissions restored. Edited and with appendix and notes of his grand-daughter Nora Barlow. Londres : Collins.

Elkin, A. (1978). Les aborigènes australiens. Dans Encyclopaedia Universalis (vol. 2) (p. 831-833).

Gould, S. (1977/1997) Le véritable père de l'homme est l'enfant. Dans Darwin et les grandes histoires de la vie. Réflexions sur I'histoire naturelle (p. 64-71). Paris : Seuil.

Gourou, P. (1963). Le déterminisme physique dans L'Esprit des lois. L'Homme, 3(3), 5-11.

Huertas, H. (2013, 29 octobre). Le billet politique d'Hubert Huertas « Taubira face au racisme : silence, on coule... » [vidéo en ligne]. France culture. http://www.franceculture.fr/emission-le-billet-politique-d-hubert-huertastaubira-face-au-racisme-silence-on-coule-2013-10-29

Huffington Post (2013, 21 octobre). Christiane Taubira comparée à un singe par une candidate FN : ouverture d'une enquête préliminaire [en ligne]. Huffington Post. http://www.huffingtonpost.fr/2013/10/21/taubira-singeenquete-preliminaire-candidate-front-national_n_4136459.html

Indigenous Film Services (2008, 13 février). The Apology [vidéo en ligne]. Reconciliation Australia. www.reconciliation.org.au. (consultable en ligne à http://www.youtube.com/watch?v=DUvPxmiudSI\&feature=player_embedded)

Milmo, C. (2007, 17 octobre). Fury at DNA pioneer's theory: Africans are less intelligent than Westerners [en ligne. The Independent. http://news.independent.co.uk/sci tech/article3067222.ece

Moisseeff, M. (1997). Subversive science, subversive practice. Transcultural psychiatry, 34(1), 95-107.

Moisseeff, M. (1999a). An Aboriginal village in South Australia. A snapshot of Davenport. Canberra (Australie) : Aboriginal Studies Press.

Moisseeff, M. (1999b). La hiérarchisation des cultures, un autre regard sur les migrations et l'exclusion sociale. Thérapie familiale, 20(3), 237-252.

Moisseeff, M. (2000). Une figure de l'altérité chez les Dentcico ou la maternité comme puissance maléfique. Dans J.-L. Jamard, E. Terray et M. Xanthakou (dir.), En substances. Textes pour Françoise Héritier (p. 471-489). Paris : Fayard.

Moisseeff, M. (2004). L'amour extraterrestre : une mythologie à méditer. Dans F. Héritier et M. Xanthakou (dir.), Corps et affects (p. 325-338). Paris : Odile Jacob.

Moisseeff, M. (2005a). Penser le métissage : Une interrogation pour les sciences sociales. L'autre, Cliniques, cultures et sociétés, 6(2), 287-304.

Moisseeff, M. (2005b). La procréation dans les mythes contemporains : une histoire de science-fiction. Anthropologie et sociétés, 29(2), 69-94.

Moisseeff, M. (2008a). Alien ou ou le retour d'un mythe polynésien. Dans J. Daniel (dir.), Le siècle de Lévi-Strauss (p. 157-164). Paris : CNRS Éditions.

Moisseeff, M. (2008b). Nous n'avons jamais été humains. Le néotène, les chimères et les robots. Dans S. Gruzinski (dir.), Planète métisse (p. 152-165). Arles : Musée du Quai Branly/Actes Sud.

Moisseeff, M. (2010). Au cœur du système hiérarchique occidental : I'évolution biologique. Dans A. Iteanu (dir.), La cohérence. Mélanges en hommage à Daniel de Coppet (p. 341-368). Paris : Éditions de la Maison des Sciences de l'Homme.

Moisseeff, M. (2011a). Grossesses extraterrestres et implant nasal : une mythologisation du biopouvoir?. Dans J. Goffette et L. Guillaud (dir.), L'Imaginaire médical dans le fantastique et la science-fiction (p. 303-316). Paris : Bragelonne.

Alterstice - Revue Internationale de la Recherche Interculturelle, vol. $4, n^{\circ} 2$ 
Moisseeff, M. (2011b). Invisible and Visible Loyalties in Racialized Contexts: A Systemic Perspective on Aboriginal Youth. Dans U. Eickelkamp (dir.), Growing Up in Central Australia: New Anthropological Studies of Aboriginal Childhood and Adolescence, (p. 239-272). Oxford, R.-U. : Berghahn Books.

Moisseeff, M. (2013). La tyrannie du choix chez les jeunes Aborigènes australiens. L'autre, Cliniques, cultures et sociétés, 14(1), 43-53.

Moisseeff, M. (2014). Payback and forward: relational responsibility as a source of weakness or strength. Dans Actes du colloque Australian Aboriginal anthropology today: critical perspectives from Europe (en ligne). Paris : Musée du Quai Branly. http://actesbranly.revues.org/568

Ndala, B. (2013, 12 septembre). Noire et ministre en Europe: quand les extrêmes-droites voient rouge, huffingtonpost.fr. Récupéré de http://www.huffingtonpost.fr/blaise-ndala/noire-ministre-europeracisme_b_3907219.html

Neville, A. (1947). Australia's Coloured Minority: Its Place in the Community. Sydney, Australie : Currawong.

O’Rourke, D. (1984). Lang Hancock father of Gina RineHart offers sterilisation as a solution to the Aboriginal problem. Dans Couldn't Be Fairer [film documentaire australien]. Consultable à partir de la page https://www.youtube.com/watch?v=h-RV9reCDbY

Rice, A. (1993/1995). L'Heure des sorcières. Paris : Laffont.

Rice, A. (1994/1996). Taltos. Paris : Laffont.

Roselmack, H. (2013, 5 novembre). La France raciste est de retour [en ligne]. Le Monde. http://www.gala.fr/I_actu/news_de_stars/harry_roselmack_la_france_raciste_est_de_retour_301842

Sironi, F. (2007). Psychopathologie des violences collectives. Paris : Odile Jacob.

Tatz, C. (1999). Genocide in Australia (AIATSIS Research Discussion Paper $n^{\circ}$ 8). Canberra (Australie) : Australian Institute of Aboriginal and Torres Strait Islander Studies.

Taylor, A.-C. (1991), Évolutionnisme. Dans P. Bonte et M. Izard (dir.), Dictionnaire de l'ethnologie et de l'anthropologie (p. 269-272). Paris : Presses universitaires de France.

Waintrater, R. (2003/2011). Sortir du génocide. Témoignage et survivance. Paris : Payot.

Wyndham, J. (1957). The Midwich Cukoos. Londres : Michael Joseph. 